\title{
The progestogen content of combined oral contraceptives and venous thromboembolic risk
}

\author{
New evidence confirms that differences exist between preparations with different progestogens
}

\author{
Philip C Hannaford Grampian Health Board chair of primary care
}

Academic Primary Care, University of Aberdeen, Aberdeen AB25 2ZD, UK

In the linked study (doi:10.1136/bmj.d6423), Lidegaard and colleagues assess the effect of different types of combined oral contraceptive, according to progestogen type and oestrogen dose, on the risk of venous thromboembolism. ${ }^{1}$

Over the past four decades, more than 25 epidemiological studies have examined the risk of venous thromboembolism in current users of combined oral contraceptives. ${ }^{2}$ All but two studies found a significantly higher risk among current users compared with non-users. The risk is probably greater during the first few months of use, before falling to a level that remains above that of non-users until the use of these contraceptives is stopped, when the excess risk rapidly disappears. Early studies examining the effects of the hormonal constituents tended to focus on the oestrogen content of particular products, although one report suggested that the progestogen content may also be important. ${ }^{3}$ Focus on the progestogen content sharpened in the mid-1990s, when several publications reported a higher risk of venous thromboembolism in users of combined oral contraceptives containing the more recently introduced progestogens, desogestrel or gestodene, compared with those using products with the older progestogen levonorgestrel. These results, replicated in subsequent studies, have been subjected to intense scientific and legal scrutiny.

Recent studies reporting a higher risk—again relative to users of levonorgestrel containing preparations - of venous thromboembolism in users of combined oral contraceptives that contain the latest progestogen, drospirenone, have also come under close scrutiny. In the largest study, ${ }^{4}$ the usual trend of declining risk after the first few months of use was absent in women using levonorgestrel containing pills. Commentators suggested that this might have been because of "left censoring bias" as a result of periods of higher risk during early use being incompletely recorded for older levonorgestrel containing pills, unlike the newly introduced drospirenone containing products. ${ }^{5}$ Thus, the observed incidence of thrombosis in the levonorgestrel using comparator group may have been artificially low, which would have exaggerated the risk in drospirenone users.

Lidegaard and colleagues report findings from a four year extension of their previous study. ${ }^{4}$ The study used four national registries to linked data about citizenship, hospital discharge diagnoses, deaths, and medicinal products. All Danish women aged 15-49 years between 1995 and 2009 were identified and those with recorded previous thrombosis, cancer, and coagulation disturbances were excluded or censored, as were those who were pregnant or who underwent hysterectomy, bilateral oophorectomy, or sterilisation. First episodes of venous thromboembolism were identified from hospital records, and events that were treated with anticoagulants for at least four weeks were considered confirmed (67\% of all identified cases). An independent assessment of 200 cases indicated that venous thromboembolism was confirmed in most women given anticoagulation. The authors calculated person time exposure to hormonal contraception using information from the national registry of medicinal products about hormonal contraceptive prescriptions redeemed between 1995 and 2009. Although the study focused on the period after the launch of drospirenone containing combined oral contraceptives in Denmark (2001-9), prescription data from 1995 were used for the calculation of duration of hormonal contraceptive use. This greatly reduced the potential for left censoring bias. Sensitivity analyses tested assumptions made in the construction of periods of exposure, with generally few differences between results.

During the eight million women years of observation, 4246 first episodes of venous thromboembolism occurred. Among the confirmed cases, and after adjustment for age, calendar year, education, and length of use, the relative risk of venous thromboembolic risk in women who used pills with desogestrel, gestodene, or drospirenone was twice that of those who used pills with levonorgestrel. The estimated average absolute risk in current users of newer pills was about 10 per 10000 women years. Although the corresponding relative risks in non-confirmed cases tended to be smaller, those for combined oral contraceptives containing gestodene or drospirenone were still significantly greater than for those containing levonorgestrel. Preparations with a newer progestogen and 20 $\mu \mathrm{g}$ of oestrogen did not have a significantly lower risk of venous thrombosis than those with the same progestogen and $30 \mu \mathrm{g}$ of oestrogen. Progestogen only oral contraceptives and the 
levonorgestrel releasing intrauterine device were not associated with an increased risk of venous thromboembolism.

A key concern with any data linkage study is the quality of information collected, often recorded during everyday practice in an unstructured way. A crucial consideration is whether there is likely to be differential recording of information among the various exposure groups, and if so, whether this is related to outcome. There is little reason to suspect that this occurred in this study. Furthermore, the similar proportion of confirmed cases in the different groups argues against differential referral or diagnostic bias as an explanation for the study's results. Information about important potential confounders-such as body mass index and family history of venous thromboembolism-is often absent, incomplete, or out of date in routine datasets. Residual confounding should therefore always be considered in the assessment of results from data linkage studies. Such effects, however, would have to be large to account for a doubling of risk.

This new study has tackled many of the concerns expressed about the earlier investigation. Although unpalatable to some, it is difficult not to conclude that combined oral contraceptives with desogestrel, gestodene, or drospirenone confer a higher risk of venous thromboembolism than those with levonorgestrel. Many clinicians will choose to minimise the risk by prescribing a combined oral contraceptive with levonorgestrel whenever possible. It is crucial, however, not to exaggerate the risk-oral contraceptives are remarkably safe and may confer important long term benefits in relation to cancer and mortality. ${ }^{67}$
Competing interests: The author has completed the ICMJE uniform disclosure form at www.icmje.org/coi_disclosure.pdf (available on request from the corresponding author) and declares: no support from any organisation for the submitted work; his academic department has received payments from Schering Plough and Wyeth Pharmaceutical for lectures and advisory board work provided by $\mathrm{PCH}$; no other relationships or activities that could appear to have influenced the submitted work.

Provenance and peer review: Commissioned; not externally peer reviewed.

1 Lidegaard Ø, Nielsen LH, Skovlund CW, Skjeldestad FE, Løkkegaard E. Risk of venous thromboembolism from use of oral contraceptives containing different progestogens and oestrogen doses: Danish cohort study, 2001-9. BMJ 2011;343:d6423.

2 Hannaford PC. Epidemiology of the contraceptive pill and venous thromboembolism. Thromb Res 2011;127(suppl 3):S30-4.

3 Inman WHW, Vessey MP, Westerholm B, Engelund A. Thromboembolic disease and the steroidal content of oral contraceptives. A report to the Committee on Safety of Drugs. BMJ 1970;2:203-9.

4 Lidegaard Ø, Løkkegaard E, Svendsen AL, Agger C. Hormonal contraception and risk of venous thromboembolism: national follow-up study. BMJ 2009;339:b2890.

5 Shapiro S, Dinger J. Risk of venous thromboembolism among users of oral contraceptives: a review of two recently published studies. J Fam Plann Reprod Health Care 2010;36:33-8.

6 Hannaford PC, Selvaraj S, Elliott AM, Angus V, Iversen L, Lee AJ. Cancer risk among oral contraceptive users: cohort data from the Royal College of General Practitioner's oral contraception study. BMJ 2007:335:651-4.

7 Hannaford PC, Iversen L, Macfarlane TV, Elliott AM, Angus V, Lee AJ. Mortality among contraceptive pill users: cohort evidence from Royal College of General Practitioners' oral contraception study. BMJ 2010;340:c927.

Cite this as: BMJ 2011;343:d6592

(c) BMJ Publishing Group Ltd 2011 